\title{
VORTICES AND SPIRALS IN THE HD 135344B TRANSITION DISK
}

\author{
N. van der Marel ${ }^{1}$, P. Cazzolettit ${ }^{2}$, P. Pinilla ${ }^{3}$, and A. Garufi ${ }^{4,5}$ \\ ${ }^{1}$ Institute for Astronomy, University of Hawaii, 2680 Woodlawn Drive, 96822 Honolulu, HI, USA; marel@hawaii.edu \\ 2 Max-Planck-Institut fur Extraterrestrische Physik, Giessenbachstrasse 1, D-85748 Garching, Germany \\ ${ }^{3}$ Leiden Observatory, P.O. Box 9513, 2300 RA Leiden, the Netherlands \\ ${ }^{4}$ ETH, Zurich, Wolfgang-Pauli-Strasse 27, 8093, Zurich, Switzerland \\ Received 2016 April 8; accepted 2016 July 21; published 2016 November 30
}

\begin{abstract}
In recent years, spiral structures have been seen in scattered light observations and signs of vortices in millimeter images of protoplanetary disks, both probably linked with the presence of planets. We present Atacama Large Millimeter/submillimeter Array Band $7(335 \mathrm{GHz}$ or $0.89 \mathrm{~mm})$ continuum observations of the transition disk HD $135344 \mathrm{~B}$ at an unprecedented spatial resolution of 0 !" 16 , using superuniform weighting. The data show that the asymmetric millimeter-dust ring seen in previous work actually consists of an inner ring and an outer asymmetric structure. The outer feature is cospatial with the end of one of the spiral arms seen in scattered light, but the feature itself is not consistent with a spiral arm due to its coradiance. We propose a new possible scenario to explain the observed structures at both wavelengths. Hydrodynamical simulations show that a massive planet can generate a primary vortex (which dissipates at longer timescales, becoming an axisymmetric ring) and trigger the formation of a second generation vortex further out. Within this scenario, the two spiral arms observed at scattered light originate from a planet at $\sim 30$ au and from the secondary vortex at $\sim 75$ au rather than a planet further out as previously reported.
\end{abstract}

Key words: instabilities - planet-disk interactions - planets and satellites: formation - protoplanetary disks

\section{INTRODUCTION}

Protoplanetary disks are the cradles of young planets, where several dynamical processes are likely involved in the planet formation process (e.g., Armitage 2011). Of particular interest are the transition disks: disks with inner millimeter-dust cavities. In the last decade, observations have revealed that some transition disks are far from axisymmetric: azimuthal asymmetries in the submillimeter continuum are thought to be dust traps, triggered by vortices acting as azimuthal pressure bumps (e.g., Birnstiel et al. 2013; Lyra \& Lin 2013; van der Marel et al. 2013). On the other hand, near-infrared scattered light observations show large spirals (e.g., Muto et al. 2012; Garufi et al. 2013; Grady et al. 2013; Avenhaus et al. 2014). Both spirals and vortices may indicate the presence of recently formed massive planets: in the case of a vortex through Rossby wave instability (RWI) at the steep edges of the gap that is carved by the planet (Lovelace et al. 1999; de Val-Borro et al. 2007) and in the case of spirals through the trigger of density waves directly by the planet (e.g., Kley \& Nelson 2012).

Alternative explanations for spiral arms in disks include RWI at the edge of a dead zone (Lyra et al. 2015), accretion from an envelope (Lesur et al. 2015) and gravitational instability (Lodato \& Rice 2004, 2005; Rice et al. 2004), though estimated disk masses generally appear to be too low for them to be self-gravitating (Williams \& Cieza 2011).

A natural question is whether there is any relation between the spiral arms observed in near-infrared scattered light (from the disk surface) and the structures seen in submillimeter emission (from the midplane). Although spiral features in submillimeter emission have been seen in two transition disks (Piétu et al. 2005; Christiaens et al. 2014), they are not entirely

\footnotetext{
5 Universidad Autonónoma de Madrid, Dpto. Física Teórica, Módulo 15 , Facultad de Ciencias, Campus de Cantoblanco, E-28049 Madrid, Spain.
}

consistent with their near-infrared counterparts. Juhász et al. (2015), Pohl et al. (2015), and Dong et al. (2015b) demonstrated that spirals generated by planet-disk interactions more likely result from changes in the vertical structure rather than the density structure, which are hard to detect in millimeter emission. On the other hand, spirals that form through gravitational instability can trap dust (Lodato \& Rice 2004; Dipierro et al. 2015; Dong et al. 2015a), resulting in millimeter continuum spirals.

In this paper, we present Atacama Large Millimeter/ submillimeter Array (ALMA) submillimeter continuum observations at a very high spatial resolution of $\mathrm{HD}^{135344 \mathrm{~B}^{6}}$ (F4 star, $d \sim 140$ pc, $\sim 8$ Myr; van Boekel et al. 2005; Grady et al. 2009), a well-studied transition disk at both optical and millimeter wavelengths. The HD 135344B disk contains a $\sim 40$ au radius dust cavity (Brown et al. 2007, 2009; Andrews et al. 2011) with a minor azimuthal asymmetry along the dust ring (Pérez et al. 2014; Pinilla et al. 2015). CO observations and scattered light indicate that gas and small grains are present inside the cavity (Pontoppidan et al. 2008; Lyo et al. 2011; Garufi et al. 2013; van der Marel et al. 2015, 2016), consistent with a scenario where a massive planet at $\lesssim 30$ au has cleared its orbit and trapped the large dust further out (Pinilla et al. 2012). Scattered light imaging reveals two major spiral arms (Muto et al. 2012; Garufi et al. 2013; Stolker et al. 2016), proposed to be linked to planet-disk interaction, with planets located at 55 and 126 au.

The new images presented in this paper show substructure in the millimeter emission to an unprecedented level, revealing a double structure, which may be responsible for triggering the spiral arms seen in the scattered light. This new interpretation has consequences for the implied location of the putative planets.

\footnotetext{
6 also known as SAO 206462.
} 

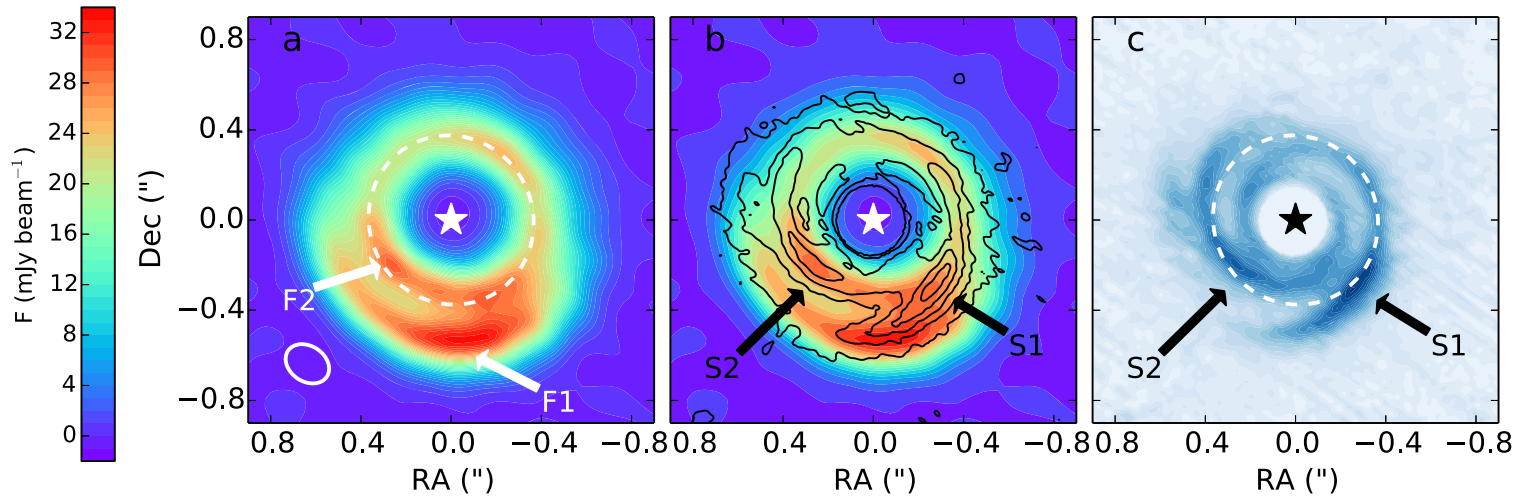

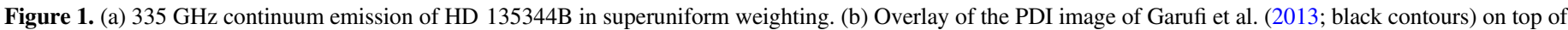

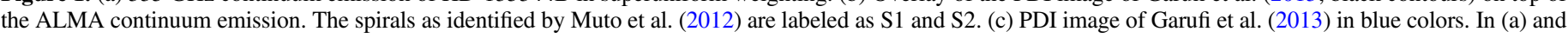
(c), the white dashed ellipse indicates the 45 au radius.

\section{OBSERVATIONS}

HD 135344B was observed in ALMA Cycle 1 program 2012.1.00158.S (PI van Dishoeck) in Band $7(\sim 335 \mathrm{GHz}$ or $896 \mu \mathrm{m})$ in the $\mathrm{C} 32-5$ configuration $(20-800 \mathrm{~m}$ baselines), previously presented in van der Marel et al. (2016) and Pinilla et al. (2015). The spectral settings and calibration are discussed in van der Marel et al. (2016). For this work, the continuum emission is reimaged using superuniform rather than briggs weighting of the observed visibilities, resulting in a smaller beam size of $0.20 \times 0$ "! 16 (Figure 1(a)). In superuniform weighting, the weights of the grid cells in the $u, v$ plane are set inversely proportional to the sampling density function, minimizing the sidelobes over an arbitrary field size, whereas briggs weighting sets the weights also inversely proportional to the noise variance of each visibility. The peak signal-to-noise ratio decreases from $210 \sigma$ (briggs) to $120 \sigma$ (superuniform) with $\sigma$ the rms level $\left(0.25 \mathrm{mJy}\right.$ beam $\left.^{-1}\right)$. We also make use of archival data of HD 135344B obtained in Polarization Differential Imaging (PDI) in the $\mathrm{K}_{s}$ band $(\sim 2.2 \mu \mathrm{m}$; Garufi et al. 2013) with VLT/NACO. The data thus obtained trace the (polarized) scattered light from the disk surface and have angular resolution of 0 " 09 .

Figure 1(a) reveals that the millimeter emission does not originate from a single dust ring with an azimuthal asymmetry, but an outer azimuthal asymmetric feature in the south (labeled F1) and an inner ring-like feature F2. With the current spatial resolution, it remains unclear whether they are connected in the southwest. These features are located at 45 and 75 au radii. The $\mathrm{F} 1$ feature is at least four times brighter than its opposite side in the north, while the $\mathrm{F} 2$ ring is almost azimuthally symmetric, with an azimuthal contrast of at most a factor of 1.2. The peak brightness temperature is $20 \mathrm{~K}$, implying that the emission is optically thick even at this wavelength $(896 \mu \mathrm{m})$.

Figure 1(b) shows the overlay of the PDI image (multiplied by the squared distance to the central star) on top of the ALMA data. The ALMA features appear to follow the spiral structure: $\mathrm{F} 1$ is at the end of the spiral S1 (as defined in Muto et al. 2012), while F2 appears to overlap with S2. The brightest part of the S1 spiral in the west is, however, not cospatial with the brightest ALMA data points, and as we will show below S1 and $\mathrm{F} 1$ are related in a different way.

In the modeling, we use the stellar position $15^{\mathrm{h}} 15^{\mathrm{m}} 48^{\mathrm{s}} .42$ $-37^{\circ} 09^{\prime} 16^{\prime \prime} 36$ as derivedfrom the ${ }^{13} \mathrm{CO}$ emission, and for the deprojection a position angle of $62^{\circ}$ and an inclination of $16^{\circ}$ (van der Marel et al. 2016).

\section{MORPHOLOGY}

In order to understand the morphology of the disk, two different models are investigated. Model 1 follows the spiral description derived by Muto et al. (2012). Model 2 consists of an inner symmetric ring and outer azimuthal asymmetry, following the morphology of the image. This double structure has been seen in certain 2D hydrodynamical simulations of planet-disk interaction, with a primary vortex at the outer edge of the planetary gap and subsequently, a second vortex external to the primary (Lobo Gomes et al. 2015).

\subsection{The Spiral Model}

We model the shape of a spiral density wave generated by a planet located at $\left(r_{\mathrm{c}}, \theta_{0}\right)$ using the analytical shape derived by Rafikov (2002). This analytical approach describes the propagation of the wave from a launching point and it is given by

$$
\begin{aligned}
\theta(r)= & \theta_{0}-\frac{\operatorname{sgn}\left(r-r_{\mathrm{c}}\right)}{h_{\mathrm{c}}} \\
& \times\left[\left(\frac{r}{r_{\mathrm{c}}}\right)^{1+\beta}\left\{\frac{1}{1+\beta}-\frac{1}{1-\alpha+\beta}\left(\frac{r}{r_{\mathrm{c}}}\right)^{-\alpha}\right\}\right. \\
& \left.-\left(\frac{1}{1+\beta}-\frac{1}{1-\alpha+\beta}\right)\right],
\end{aligned}
$$

where $h_{\mathrm{c}}$ is the disk scale-height at $r=r_{\mathrm{c}}$, disk angular velocity of $\Omega(r) \propto r^{-\alpha}$, and sound speed $c(r) \propto r^{-\beta}$. Equation (1) has been used to fit spiral arms observed in scattered light (Muto et al. 2012; Benisty et al. 2015), though the approximations assumed to derive Equation (1) may fail for massive planets ( $1_{1} M_{\text {Jup }}$, Zhu et al. 2015). This linear implementation results in one spiral for one planet, while in the nonlinear case, one planet can generate one or more spirals (Dong et al. 2015b; Zhu et al. 2015).

For Model 1, we fit Equation (1) to the position of the maxima of F2. For this purpose, we select the pixels of the S1 arm, masking out the inner ring. We also set $\alpha=1.5$ (Keplerian rotation) and $\beta=0.45$ (from the temperature profile in van der Marel et al. 2016). The value of $h_{\mathrm{c}}$ is not well constrained by any model of the system, and at the radii of interest it ranges between 0.08 and 0.16 (e.g., Andrews et al. 2011; Carmona et al. 2014; van der Marel et al. 2016). 

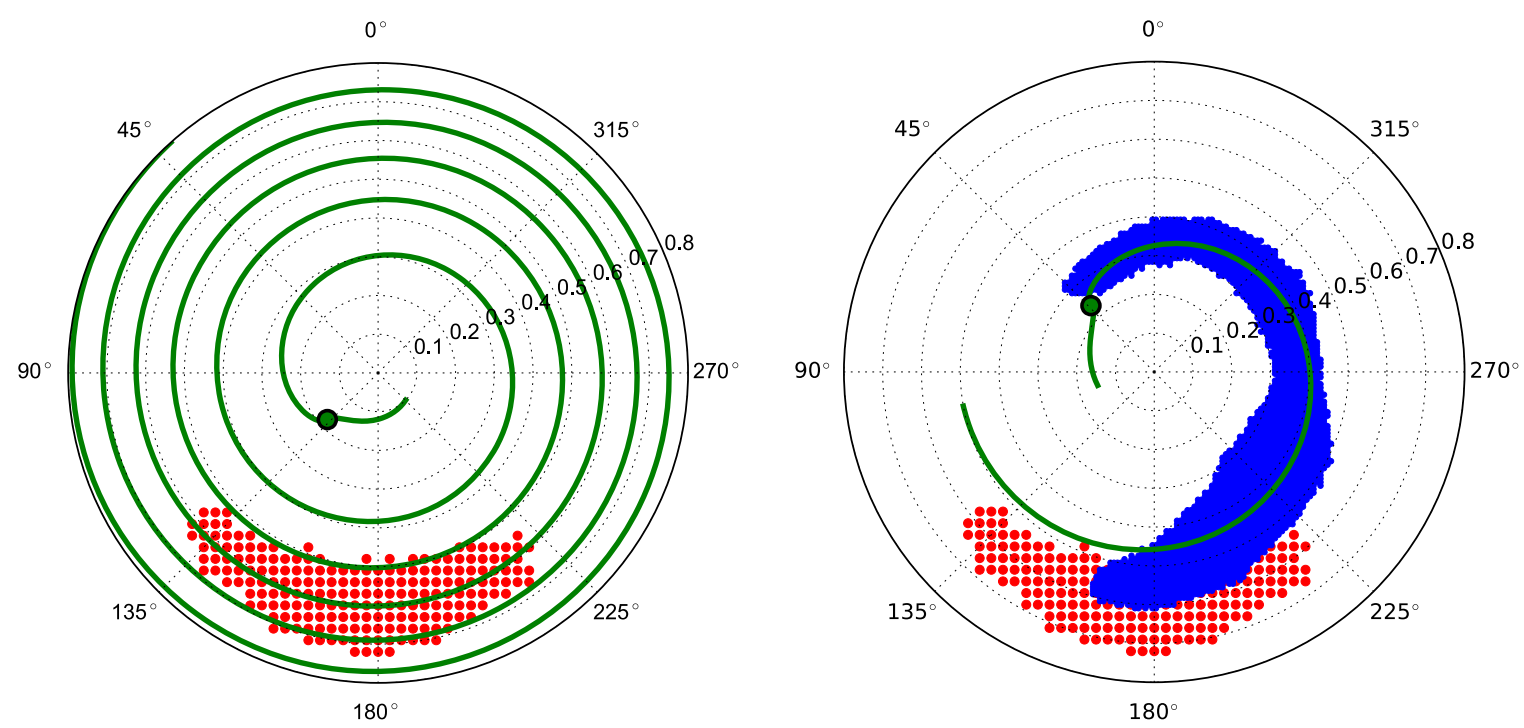

Figure 2. Left: best fit of the ALMA continuum F1 feature (red dots) to the spiral model (green). This fit is unrealistic, as density waves are damped efficiently and the fourth winding would no longer be visible. Right: overlay of the brightest data points of ALMA continuum (red dots) and the S1 feature in the scattered light data (blue dots, Garufi et al. 2013), both deprojected. The green line shows our best-fit spiral to the blue data points, with $\left(r_{c}, \theta_{0}\right)=\left(0^{\prime \prime} 24,134^{\circ}\right)$. This figure shows that F2 does not follow the spiral arm seen in scattered light.

Therefore, we fix the scale-height value to the average $h_{\mathrm{c}}=0.12$, so only two free parameters remain for the fit: $r_{\mathrm{c}}$ and $\theta_{0}$, which characterize the launching position of the spiral.

We adopt an Orthogonal Distance Regression fitting procedure, that searches for the curve that minimizes the sum of the distances to the data points orthogonally to the curve itself, thus assuming an observational error on both $\theta$ and $r$ in Equation (1). We assume the uncertainty on the positions of the maxima to be equal to the FWHM of the beam. Finally, each data point is weighted proportional to the corresponding pixel intensity.

The fit in Figure 2 (left) shows that the F1 structure is mostly coradial and hence the spiral launching position has to be very close to the central star $\left(r_{\mathrm{c}}<0\right.$." 2$)$ : the spiral pitch angle is close to $0^{\circ}$. In such a scenario, F1 would be part of the fourth spiral winding. However, the density waves after the first spiral winding are damped very efficiently due to the disk viscosity and pressure torque (Baruteau et al. 2014) and therefore this scenario is unrealistic to explain the observed azimuthal structure. Figure 2 (right) shows that the ALMA continuum does not follow the best fit to the spiral arm in scattered light (blue dots).

\subsection{The Ring Plus Asymmetry Model}

Model 2 describes the structure as a combination of a ring (F2) with a azimuthal asymmetry (F1). This model assumes that the asymmetry may originate from a vortex, using the vortex prescription by Lyra \& Lin (2013) of a Gaussian in the radial and azimuthal direction:

$$
F(r, \theta)=F_{\mathrm{v}} e^{-\left(r-r_{\mathrm{v}}\right)^{2} / 2 \sigma_{\mathrm{r}, \mathrm{v}}^{2}} e^{-\left(\theta-\theta_{\mathrm{v}}\right)^{2} / 2 \sigma_{\theta, v}^{2},}
$$

where $F_{\mathrm{v}}$ is the flux density at $\left(r_{\mathrm{v}}, \theta_{\mathrm{v}}\right), r_{\mathrm{v}}$ and $\theta_{\mathrm{v}}$ (east of north) are the radial and azimuthal position of the asymmetry respectively, and $\sigma_{\mathrm{r}, \mathrm{v}}$ and $\sigma_{\theta, \mathrm{v}}$ are the radial and azimuthal width of the asymmetry. F2, on the other hand, is modeled as a Gaussian ring,

$$
F(r, \theta)=F_{\mathrm{r}} e^{-\left(r-r_{\mathrm{r}}\right)^{2} / 2 \sigma_{\mathrm{r}, \mathrm{r}}^{2}},
$$

where $F_{\mathrm{r}}$ is the flux density at $r_{\mathrm{r}}$, and where $r_{\mathrm{r}}$ and $\sigma_{\mathrm{r}, \mathrm{r}}$ are the radial position and width of the ring respectively.

Our model, therefore, has eight free parameters (five for the asymmetry and three for the ring model), and we fit it to the image using the MCMC python package emcee. The chains from the fit show good convergence for all the free parameters, and the best-fit parameters are

\begin{tabular}{ll}
\hline \hline$F_{\mathrm{v}}$ & $1.44 \pm 1.6 \times 10^{-3} \mathrm{mJy} /$ pixel \\
$r_{\mathrm{v}}$ & $80.7 \pm 0.005 \mathrm{au}\left(00^{\prime \prime} 58 \pm 33^{\prime \prime} 3 \times 10^{-5}\right)$ \\
$\sigma_{\mathrm{r}, \mathrm{v}}$ & $6.3 \pm 0.008 \mathrm{au}\left(0 !^{\prime \prime} 045 \pm 5 !^{\prime \prime} 7 \times 10^{-5}\right)$ \\
$\theta_{\mathrm{v}}$ & $172^{\circ} \pm 0.02$ \\
$\sigma_{\theta, \mathrm{v}}$ & $57^{\circ} \pm 0.02$ \\
$F_{\mathrm{r}}$ & $0.96 \pm 6.1 \times 10^{-4} \mathrm{mJy} /$ pixel \\
$r_{\mathrm{r}}$ & $51.3 \pm 0.004 \mathrm{au}\left(0 ! .37 \pm 22^{\prime \prime} 8 \times 10^{-5}\right)$ \\
$\sigma_{\mathrm{r}, \mathrm{r}}$ & $8.1 \pm 0.007 \mathrm{au}\left(0 !^{\prime \prime} 058 \pm 4 . ! 8 \times 10^{-5}\right)$ \\
\hline
\end{tabular}

The errors from the MCMC calculations are much smaller than the spatial uncertainty from the observations, which is typically $\sim 10 \%$ of the beam size (i.e., $2-3$ au). Figure 3 shows the comparison between the convolved model and the observations. The best fit was simulated onto the observed visibilities, and no significant differences were found with the convolved image. Some residuals are still present, mostly due to the asymmetry in the inner ring, but at the $10 \%$ level of the original flux. The radius of the vortex is at a larger radius than found by earlier fitting of the millimeter data (Pérez et al. 2014; Pinilla et al. 2015), which could be due to their central position being 11 au away from this study.

\section{DISCUSSION AND CONCLUSIONS}

The F1 feature is not consistent with the spiral arm prescription, but it can be described as a ring ( $\sim 50 \mathrm{au})$ with an asymmetry at $\sim 80 \mathrm{au}$. Therefore, we propose a new alternative scenario for this disk to explain the structure of both millimeter and scattered light data. The millimeter geometry is consistent with a model from Lobo Gomes et al. (2015), showing that a planet generates a pressure bump at 50 au (F2), which triggers a second generation vortex at 80 au 

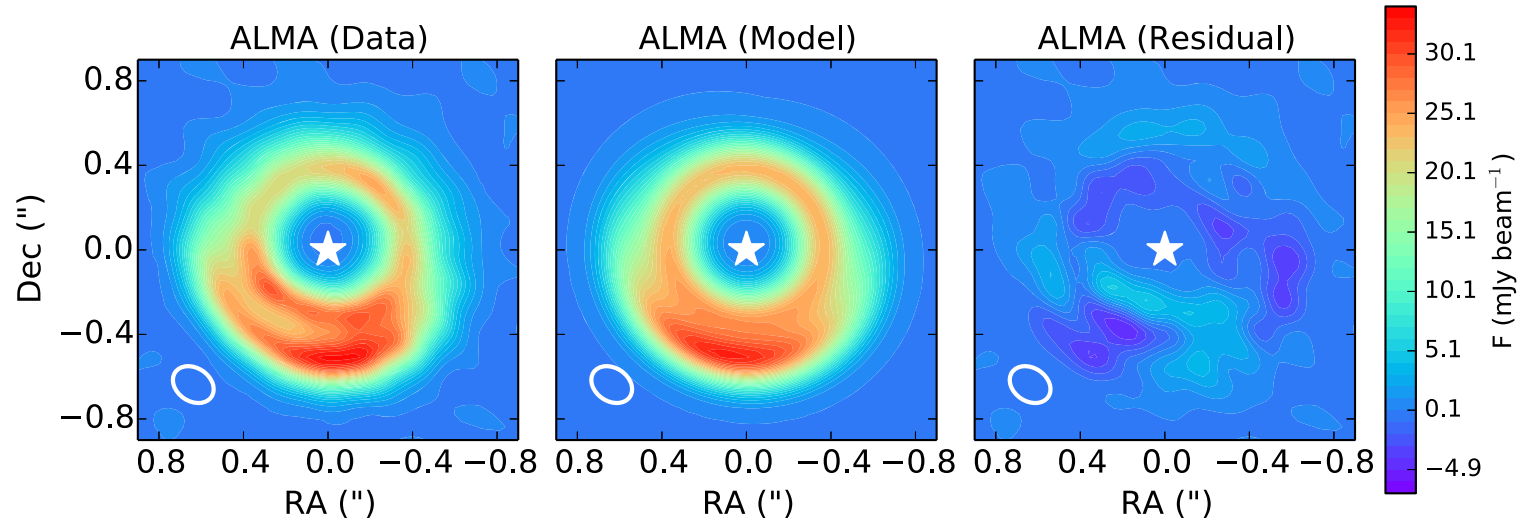

Figure 3. Best-fit model for a ring in combination with a vortex (2D Gaussian) for the ALMA continuum data. Modeling has been performed in the uv plane.
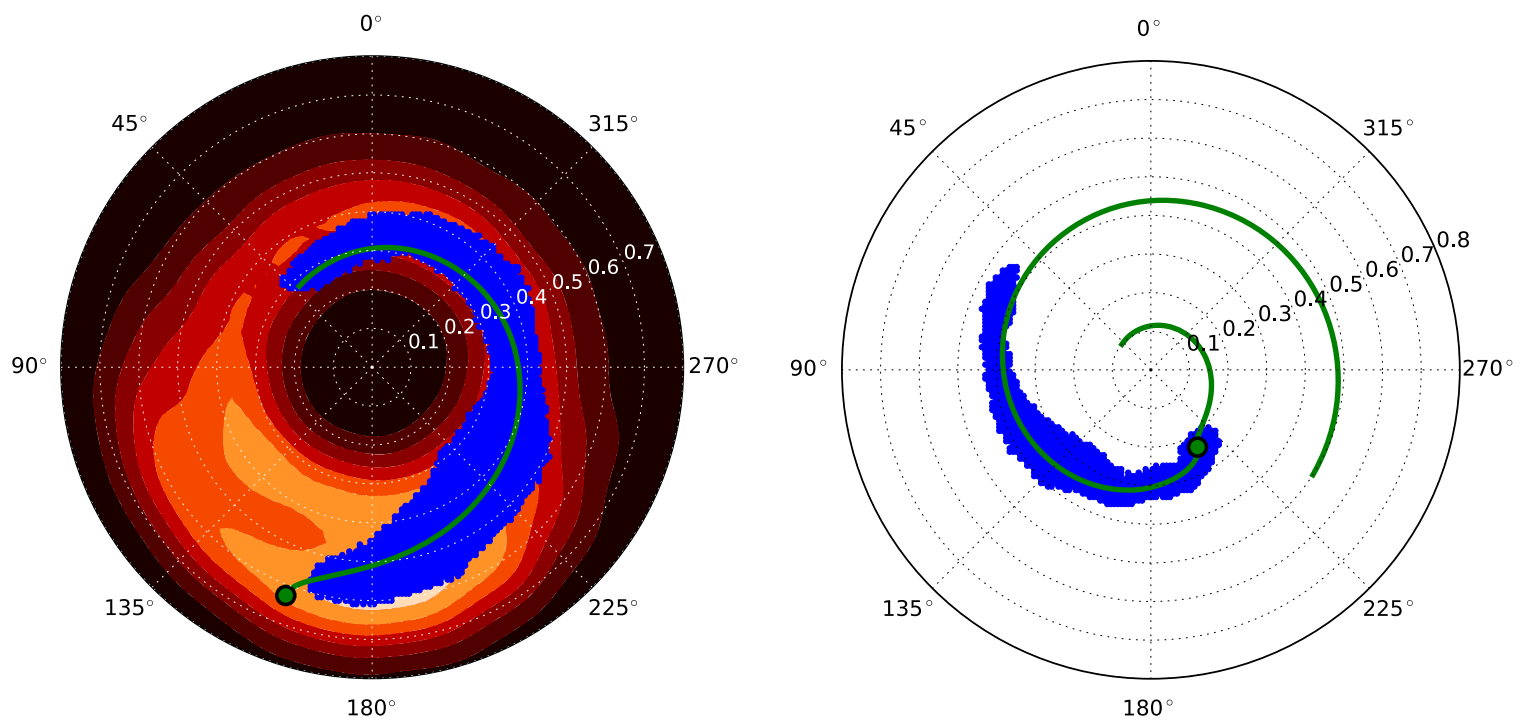

Figure 4. Left: best fit for the S1 spiral in the scattered light data (deprojected), with a starting point inside the vortex. Overlaid on the ALMA image (colors), the blue dots indicate the data points of the PDI S1 feature with the central ring masked out, and the green line the best-fit spiral, with launching point $\left(r_{c}, \theta_{0}\right)=\left(00^{\prime \prime} 62,170^{\circ}\right)$ marked as a circle. Right: best fit for the S2 spiral, with a starting point in the inner part of the disk. The blue dots are the brightest points of the PDI S2 feature and the green line the best-fit spiral, with launching point $\left(r_{c}, \theta_{0}\right)=\left(0,23,211^{\circ}\right)$ marked as a circle.

(F1). The cavity radius of the gas and small grains (Garufi et al. 2013; van der Marel et al. 2016) suggests the presence of a massive planet at 30 au. A millimeter-dust ring at 50 au (F2) is consistent with this scenario, as the dust is trapped further out than the gas gap edge (Pinilla et al. 2012).

The ALMA and PDI data trace different grain size populations and disk heights, possibly driven by different mechanisms. However, it is striking that F1 coincides with the edge of the S1 arm. We propose that the S1 is triggered by a vortex that has created the dust asymmetry F1, since vortices can be massive enough to launch their own density waves in a disk when self-gravity is included in hydrodynamical models (e.g., Baruteau \& Zhu 2016). Only a lower limit to the mass of the F1 feature can be set as the emission is partially optically thick: with a total flux of $200 \mathrm{mJy}$ and an ISM gas-to-dust ratio of 100 , the total mass is $>16 M_{\text {Jup }}$ (using $M_{\mathrm{gas}}=0.08 * F_{\nu}(d / 140 \mathrm{pc})^{2} M_{\mathrm{Jup}}$, Cieza et al. 2008). The outer extent of S1 (outside the vortex) remains undetectable in the PDI image due to the lower brightness in the outer disk.

Muto et al. (2012) find a best fit for the launching point of S1 at $r_{\mathrm{c}}=0 . \prime 39(55 \mathrm{au})$ at $\theta_{0}=204^{\circ}$, but with a large confidence interval (see Figure 5 in Muto et al.). Fitting the S1 spiral with an initial guess close to the center of the vortex results in the fit in Figure 4(a) with $r_{c}, \theta_{0}=00^{\prime \prime} 6,180^{\circ}(84 \mathrm{au})$ and $h_{\mathrm{c}}=0.08$. This launching point does not coincide exactly with the center of F1, though there is a large uncertainty due to the unknown scale height at this location. Furthermore, ALMA continuum observations trace the millimeter-dust, whose center may not coincide with the gas vortex (Baruteau \& Zhu 2016), and the vortex can be a large scale structure where the center of mass may not be well represented by a single location, contrary to a planet.

On the other hand, the $\mathrm{S} 2$ spiral was best fit by Muto et al. (2012) for $r_{c}, \theta_{0}=0 . \prime 9(126 \mathrm{au}), 353^{\circ}$, but we find that it can also be fit with a launching point in the inner part of the disk for $r_{c}, \theta_{0}=0$ "! $23\left(32 \mathrm{au}\right.$ ), $211^{\circ}$ (Figure 4(b)). The launching point of S2 would be a massive planet, just inside the gas cavity radius (van der Marel et al. 2016). Stolker et al. (2016) finds a best fit for the S2 launching point to the VLT/ SPHERE data slightly further in, at $r_{c}, \theta_{0}=0$ !" 15 (21 au), $247^{\circ}$.

We propose that the combination of the scattered light and the millimeter observations is consistent with the following sequence of events (see Figure 5). 


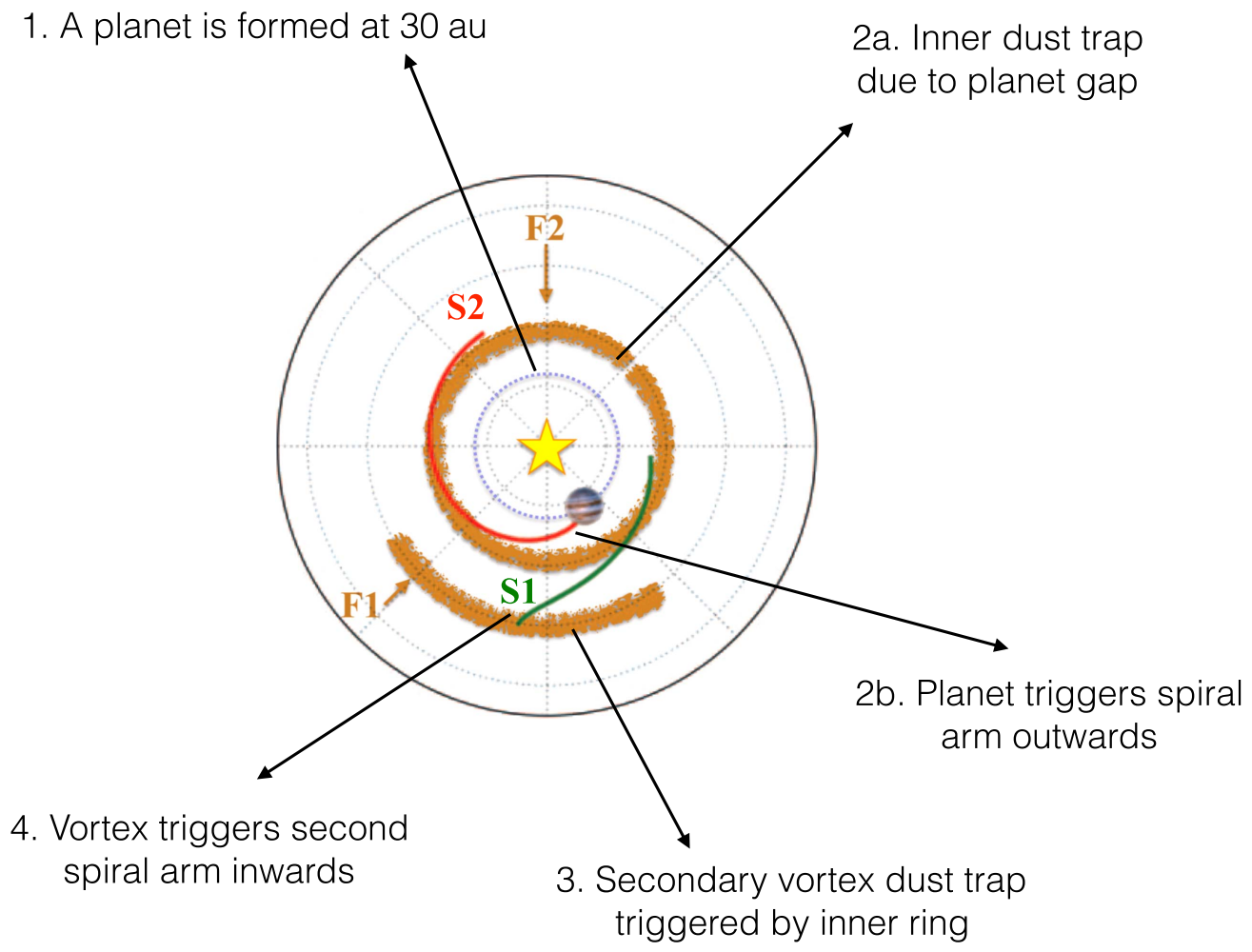

Figure 5. Cartoon explaining the proposed scenario.

1. A massive planet is formed at an $\sim 30$ au radius.

2. The planet triggers a spiral density wave outwards (PDI S2 feature).

3. The planet clears its orbit in the gas (CO observations) and creates a radial pressure bump at its edge where millimeter-dust gets trapped (ALMA continuum F2 feature).

4. The pressure bump creates an effective $\alpha$ viscosity that is large enough to induce accretion, depleting the gas and inducing a second pressure bump farther out. The second pressure bump triggers RWI, forms a vortex, and traps the millimeter-dust asymmetrically (ALMA continuum F1 feature).

5. The outer vortex triggers a spiral density wave inwards (PDI S1 feature).

This scenario can potentially explain both PDI and millimeter observations. Hydrodynamical models of gas and dust, including self-gravity, are required to check whether our proposed scenario can instead quantitatively explain the observed structures of HD 135344B.

One of the major uncertainties in the scenario are the fits to the locations of the launching points. The reason is that the scattered light data are mainly sensitive to changes in the scale height and, therefore, the observed scattered light is significantly affected by geometric parameters. The observed spirals form only the illuminated inner part of a surface change. Also, the inner disk region may shadow the outer part and thus alter the intrinsic disk scale-height distribution. In particular, the azimuthal angle of the continuum ALMA feature coincides with the brighter part of the closer-in S2 spiral and therefore, S2 may be casting a shadow on part of S1, affecting the fit of the launching points.
Another caveat is the symmetry of the two spiral arms at the time of observation, suggesting a common nature such as that proposed by Dong et al. (2015b), who demonstrate the trigger of two symmetric spiral arms by a single planet at $100 \mathrm{au}$. As this planet has remained undetected, this scenario cannot be confirmed. On the other hand, if there are instead two launching points (32 and $86 \mathrm{au}$ ), the two spirals would have distinct angular velocities and their symmetric appearance is fortuitous, making the scenario less probable. The orbital period of the 32 au point is only 143 years, implying a $2.5 /$ year angular shift. Repeating the scattered light observations in five years should clearly reveal the motion of this arm. If the asymmetry is indeed related to a vortex, an azimuthal shift of $\sim 0$ ! $1\left(6^{\circ}\right)$ in the millimeter continuum (measurable at 0 .! 2 resolution) is detectable after 10 years.

The scenario is an example of triggered planet formation, where the formation of a first planet can induce dust growth and potentially further planet formation in the outer disk.

We are grateful to E.F. van Dishoeck, M. Tazzari, S. Facchini, and T. Muto for useful discussions. N.M. is supported by the Beatrice Watson Parrent Fellowship. Astrochemistry in Leiden is supported by NOVA, KNAW, and EU A-ERC grant 291141 CHEMPLAN. This paper makes use of the following ALMA data: ADS/JAO.ALMA/2012.1.00158. S. ALMA is a partnership of ESO (representing its member states), NSF (USA), and NINS (Japan), together with NRC (Canada) and NSC and ASIAA (Taiwan), in cooperation with the Republic of Chile. The Joint ALMA Observatory is operated by ESO, AUI/NRAO, and NAOJ.

Facility: ALMA. 


\section{REFERENCES}

Andrews, S. M., Wilner, D. J., Espaillat, C., et al. 2011, ApJ, 732, 42 Armitage, P. J. 2011, ARA\&A, 49, 195

Avenhaus, H., Quanz, S. P., Schmid, H. M., et al. 2014, ApJ, 781, 87

Baruteau, C., Crida, A., Paardekooper, S.-J., et al. 2014, in Protostars and Planets VI, ed. H. Beuther et al. (Tuscon, AZ: Univ. Arizona Press)

Baruteau, C., \& Zhu, Z. 2016, MNRAS, 458, 3927

Benisty, M., Juhasz, A., Boccaletti, A., et al. 2015, A\&A, 578, L6

Birnstiel, T., Dullemond, C. P., \& Pinilla, P. 2013, A\&A, 550, L8

Brown, J. M., Blake, G. A., Dullemond, C. P., et al. 2007, ApJL, 664, L107

Brown, J. M., Blake, G. A., Qi, C., et al. 2009, ApJ, 704, 496

Carmona, A., Pinte, C., Thi, W. F., et al. 2014, A\&A, 567, A51

Christiaens, V., Casassus, S., Perez, S., van der Plas, G., \& Ménard, F. 2014, ApJL, 785, L12

Cieza, L. A., Swift, J. J., Mathews, G. S., \& Williams, J. P. 2008, ApJL, 686, L115

de Val-Borro, M., Artymowicz, P., D’Angelo, G., \& Peplinski, A. 2007, A\&A, 471, 1043

Dipierro, G., Pinilla, P., Lodato, G., \& Testi, L. 2015, MNRAS, 451, 5493

Dong, R., Hall, C., Rice, K., \& Chiang, E. 2015a, ApJ, 812, 32

Dong, R., Zhu, Z., Rafikov, R. R., \& Stone, J. M. 2015b, ApJL, 809, L5

Garufi, A., Quanz, S. P., Avenhaus, H., et al. 2013, A\&A, 560, A105

Grady, C. A., Muto, T., Hashimoto, J., et al. 2013, ApJ, 762, 48

Grady, C. A., Schneider, G., Sitko, M. L., et al. 2009, ApJ, 699, 1822

Juhász, A., Benisty, M., Pohl, A., et al. 2015, MNRAS, 451, 1147

Kley, W., \& Nelson, R. P. 2012, ARA\&A, 50, 211

Lesur, G., Hennebelle, P., \& Fromang, S. 2015, A\&A, 582, L9

Lobo Gomes, A., Klahr, H., Uribe, A. L., Pinilla, P., \& Surville, C. 2015, ApJ, 810,94
Lodato, G., \& Rice, W. K. M. 2004, MNRAS, 351, 630

Lodato, G., \& Rice, W. K. M. 2005, MNRAS, 358, 1489

Lovelace, R. V. E., Li, H., Colgate, S. A., \& Nelson, A. F. 1999, ApJ, 513,805

Lyo, A.-R., Ohashi, N., Qi, C., Wilner, D. J., \& Su, Y.-N. 2011, AJ, 142,151

Lyra, W., \& Lin, M.-K. 2013, ApJ, 775, 17

Lyra, W., Turner, N. J., \& McNally, C. P. 2015, A\&A, 574, A10

Muto, T., Grady, C. A., Hashimoto, J., et al. 2012, ApJL, 748, L22

Pérez, L. M., Isella, A., Carpenter, J. M., \& Chandler, C. J. 2014, ApJL, 783, L13

Piétu, V., Guilloteau, S., \& Dutrey, A. 2005, A\&A, 443, 945

Pinilla, P., Benisty, M., \& Birnstiel, T. 2012, A\&A, 545, A81

Pinilla, P., van der Marel, N., Perez, L., et al. 2015, A\&A, 584, A16

Pohl, A., Pinilla, P., Benisty, M., et al. 2015, MNRAS, 453, 1768

Pontoppidan, K. M., Blake, G. A., van Dishoeck, E. F., et al. 2008, ApJ, 684, 1323

Rafikov, R. R. 2002, ApJ, 569, 997

Rice, W. K. M., Lodato, G., Pringle, J. E., Armitage, P. J., \& Bonnell, I. A. 2004, MNRAS, 355, 543

Stolker, T., Dominik, C., Avenhaus, H., et al. 2016, arXiv:1603.00481

van Boekel, R., Dullemond, C. P., \& Dominik, C. 2005, A\&A, 441, 563

van der Marel, N., van Dishoeck, E. F., Bruderer, S., et al. 2013, Sci, 340,1199

van der Marel, N., van Dishoeck, E. F., Bruderer, S., et al. 2016, A\&A, 585, A58

van der Marel, N., van Dishoeck, E. F., Bruderer, S., Pérez, L., \& Isella, A. 2015, A\&A, 579, A106

Williams, J. P., \& Cieza, L. A. 2011, ARA\&A, 49, 67

Zhu, Z., Dong, R., Stone, J. M., \& Rafikov, R. R. 2015, ApJ, 813, 88 